\title{
Fully Dynamic Spectral Vertex Sparsifiers and Applications
}

\author{
David Durfee* \\ Georgia Institute of Technology, USA \\ Gramoz Goranci ${ }^{\ddagger}$ \\ University of Vienna \\ Faculty of Computer Science, Austria
}

\author{
$\mathrm{Yu} \mathrm{Gao}{ }^{\dagger}$ \\ Georgia Institute of Technology, USA \\ Richard Peng \\ Georgia Institute of Technology, USA
}

\begin{abstract}
We study dynamic algorithms for maintaining spectral vertex sparsifiers of graphs with respect to a set of terminals $T$ of our choice. Such objects preserve pairwise resistances, solutions to systems of linear equations, and energy of electrical flows between the terminals in $T$. We give a data structure that supports insertions and deletions of edges, and terminal additions, all in sublinear time. We then show the applicability of our result to the following problems.

(1) A data structure for dynamically maintaining solutions to Laplacian systems $\mathbf{L x}=\mathbf{b}$, where $\mathbf{L}$ is the graph Laplacian matrix and $\mathbf{b}$ is a demand vector. For a bounded degree, unweighted graph, we support modifications to both $\mathbf{L}$ and $\mathbf{b}$ while providing access to $\epsilon$-approximations to the energy of routing an electrical flow with demand $\mathbf{b}$, as well as query access to entries of a vector $\tilde{\mathbf{x}}$ such that $\left\|\tilde{\mathbf{x}}-\mathbf{L}^{\dagger} \mathbf{b}\right\|_{\mathbf{L}} \leq \epsilon\left\|\mathbf{L}^{\dagger} \mathbf{b}\right\|_{\mathbf{L}}$ in $\tilde{O}\left(n^{11 / 12} \epsilon^{-5}\right)$ expected amortized update and query time.
\end{abstract}

(2) A data structure for maintaining fully dynamic All-Pairs Effective Resistance. For an intermixed sequence of edge insertions, deletions, and resistance queries, our data structures returns $(1 \pm \epsilon)$ approximation to all the resistance queries against an oblivious adversary with high probability. Its expected amortized update and query times are $\tilde{O}\left(\min \left(m^{3 / 4}, n^{5 / 6} \epsilon^{-2}\right) \epsilon^{-4}\right)$ on an unweighted graph, and $\tilde{O}\left(n^{5 / 6} \epsilon^{-6}\right)$ on weighted graphs.

The key ingredients in these results are (1) the intepretation of Schur complement as a sum of random walks, and (2) a suitable choice of terminals based on the behavior of these random walks to make sure that the majority of walks are local, even when the graph itself is highly connected and (3) maintenance of these local walks and numerical solutions using data structures.

\footnotetext{
*This material is based upon work supported by the National Science Foundation under Grant No. 1718533.

$\dagger$ The author was supported by the ACO program at the Georgia Institute of Technology. ¥This work was done while visiting the Georgia Institute of Technology. The research leading to these results has received funding from the Marshall Plan Foundation and the European Research Council under the European Union's Seventh Framework Programme (FP/2007-2013) / ERC Grant Agreement no. 340506.
}

Permission to make digital or hard copies of all or part of this work for personal or classroom use is granted without fee provided that copies are not made or distributed for profit or commercial advantage and that copies bear this notice and the full citation on the first page. Copyrights for components of this work owned by others than ACM must be honored. Abstracting with credit is permitted. To copy otherwise, or republish, to post on servers or to redistribute to lists, requires prior specific permission and/or a fee. Request permissions from permissions@acm.org.

STOC '19, June 23-26, 2019, Phoenix, AZ, USA

(c) 2019 Association for Computing Machinery.

ACM ISBN 978-1-4503-6705-9/19/06 . \$15.00

https://doi.org/10.1145/3313276.3316379
These results together represent the first data structures for maintain key primitives from the Laplacian paradigm for graph algorithms in sublinear time without assumptions on the underlying graph topologies. The importance of routines such as effective resistance, electrical flows, and Laplacian solvers in the static setting make us optimistic that some of our components can provide new building blocks for dynamic graph algorithms.

\section{CCS CONCEPTS}

- Theory of computation $\rightarrow$ Dynamic graph algorithms.

\section{KEYWORDS}

Dynamic graph algorithms, Schur complement, effective resistance, graph Laplacian

ACM Reference Format:

David Durfee, Yu Gao, Gramoz Goranci, and Richard Peng. 2019. Fully Dynamic Spectral Vertex Sparsifiers and Applications. In Proceedings of the 51st Annual ACM SIGACT Symposium on the Theory of Computing (STOC '19), June 23-26, 2019, Phoenix, AZ, USA. ACM, New York, NY, USA, 12 pages. https://doi.org/10.1145/3313276.3316379

\section{INTRODUCTION}

Problems arising from analyzing and understanding graph structures have motivated the development of many powerful tools for storing and compressing graphs and networks. One such tool that has received a considerable amount of attention over the past two decades is graph sparsification [8, 9]. Roughly speaking, a graph sparsifier is a "compressed" version of a large input graph that preserves important properties like distance information [51], cut value [9] or graph spectrum [61]. Graph Sparsifiers fall into two main categories: edge sparsifiers, which are graphs that reduce the number of edges, and vertex sparsifiers, which are graphs that reduce the number of vertices. Both categories have many applications in approximation algorithms [20, 54], machine learning [41, 42, 66], and most recently efficient graph algorithms [33, 43, 58, 62]. While edge sparsifiers have played an instrumental role in obtaining nearly linear time algorithms [8], their practical applicability is somewhat limited due to the fact most of the large networks are already sparse. On the other hand, vertex sparsifiers address the "real" compression of large networks by reducing the number of vertices.

While vertex sparisifers in general are significantly more difficult to generate $[12,45,46]$, a notable exception is vertex sparsifiers for quadratic minimization problems, otherwise known as Schur complements. Concretely, given an undirected, weighted graph $G$, a subset of terminal vertices $T$ and its corresponding Laplacian 
matrix, a graph $H$ with $V(H)=T$ is a vertex resistance sparsifier of $G$ with respect to $T$ if the Laplacian matrix of $H$ is obtained by the Schur complement of the Laplacian of $G$ with respect to $T$. Schur complement is a central concept in physics and linear algebra with a wide range of applications including multi-grid solvers, Markov chains and finite-element analysis [15], and has also recently found extensive applications in graph algorithms [16, 17, 36, 37, 56, 57].

Most of the massive graphs in the real world, such as social networks, the web graph, are subject to frequent changes over time. This dynamic behavior of graphs has been studied for several important graph problems, where the basic idea is to maintain problem solutions as graphs undergo edge insertions and deletions in time faster than recomputing the solution from scratch. Dynamic graph algorithms have also been formulated for many problems that involve edge sparsifiers [24, 30, 32, 65], as well important variants of edge sparsifiers themselves, including minimum spanning trees [31, 47, 48, 67], spanners [7], spectral sparsifiers [2], and low-stretch spanning trees [25]. However, despite the increasing importance of high quality vertex sparsifiers in graph algorithms, very little is known about their maintenance in the dynamic setting.

In this paper we give the first non-trival dynamic algorithms for maintaining Schur complements of general graphs with respect to a set of terminal of our choice. Our data-structure maintains at any point of time a $(1 \pm \epsilon)$ approximation to the Schur complement while supporting insertions and deletions of edges, and arbitrary vertex additions to the terminal set. To the best of our knowledge, prior dynamic Schur complement algorithms were only known for minor-free graphs [22, 23].

Lemma 1.1. Given an error parameter $\epsilon>0$, an unweighted undirected multi-graph $G=(V, E)$ with $n$ vertices, $m$ edges, a subset of terminal vertices $T^{\prime}$ and a parameter $\beta \in(0,1)$ such that $\left|T^{\prime}\right|=O(\beta m)$, there is a data-structure DyNAMICSC $\left(G, T^{\prime}, \beta\right)$ for maintaining a graph $\tilde{H}$ with $\mathbf{L}_{\tilde{H}} \approx_{\epsilon} \operatorname{SC}(G, T)$ for some $T$ with $T^{\prime} \subseteq T$, $|T|=O(\beta m)$, while supporting $O(\beta m)$ operations in the following running times:

- Initialize $\left(G, T^{\prime}, \beta\right)$ : Initialize the data-structure, in $\tilde{O}\left(m \beta^{-2} \epsilon^{-4}\right)^{1}$ expected amortized time.

- INSERT(u,v): Insert the edge $(u, v)$ to $G$ in Õ (1) amort. time.

- $\operatorname{DeLETE}(u, v)$ : Delete the existing edge $(u, v)$ from $G$ in $\tilde{O}(1)$ amortized time.

- AdDTerminal(u): Add u to $T^{\prime}$ in Õ(1) amortized time.

Our guarantees hold against an oblivious adversary.

Our algorithm extends to weighted graphs, albeit with slightly larger running time guarantees. Concretely we give an algorithm that maintains an approximate Schur Complement with $\tilde{O}\left(m \beta^{-4} \epsilon^{-4}\right)$ expected amortized time for initializing the data-structure, and $\tilde{O}(1)$ amortized time for the remaining operations.

The key algorithmic components behind the result in unweighted graphs are (1) the interpretation of Schur complement as a sum of random walks and (2) randomly picking a terminal vertex subset onto which the vertex resistance sparsifiers is constructed. Specifically, in a novel way we combine random walk based methods for generating resistance vertex sparsifiers [17] with results in combinatorics that bound the speed at which such walks spread among

\footnotetext{
${ }^{1}$ We use $\tilde{O}(f)$ to denote $O(f \cdot \operatorname{poly}(\log n))$ for a function $f$.
}

distinct edges [5]. Our result in the weighted case essentially follows the same idea except that the speed at which random walks visit distinct edges in weighted networks could be very slow. To control this, we show how to efficiently sample a distinct edge without the need to simulate every step of the walk. This interacts well with other parts of our data structure and leads to comparable running time guarantees.

We show the applicability of our dynamic Schur complement to two cornerstone problems in graph Laplacian literature, namely dynamic Laplacian solver [62] and dynamic All-Pairs Effective Resistances [60].

Solving linear systems lies at the heart of many problems arising in scientific computing, numerical linear algebra, optimization and computer science. An important subclass of linear systems are Laplacian systems, which arise in many natural contexts, including computation of voltages and currents in electrical network. Solving Laplacian system has received increasing attention over the past years after the breakthrough work of Spielman and Teng [62] who gave the first near-linear time algorithm. Motivated by fast Laplacian solvers in different model of computations [4,53], we initiate the study of algorithms for dynamically solving Laplacian systems. Concretely, given a graph Laplacian $\mathrm{L} \in \mathbb{R}^{n \times n}$ and a vector $\mathbf{b} \in \mathbb{R}^{n}$ in the range of $\mathbf{L}$, the goal is to maintain an $\mathbf{x}$ such that $\mathbf{L x}=\mathbf{b}$, while off-diagonals of $\mathbf{L}$ and the entries of $\mathbf{b}$ change over time. To allow for sub-linear query times, here we focus on querying one (or a few) coordinates of $\mathbf{x}$. Formally, given any index $u \in\{1, \ldots, n\}$, the goal is to output $\tilde{\mathbf{x}}(u)$ for some approximation $\tilde{\mathbf{x}}$ of $\mathbf{L}^{\dagger} \mathbf{b}$. Our contribution is the first sub-linear dynamic Laplacian solver in bounded degree graphs.

Theorem 1.2. For any given error threshold $m^{-1}<\epsilon<1$, there is a data-structure for maintaining an unweighted, undirected bounded degree multi-graph $G=(V, E)$ with $n$ vertices, $m$ edges and a vector $\mathbf{b} \in \mathbb{R}^{n}$ that supports the following operations in $\tilde{O}\left(n^{11 / 12} \epsilon^{-5}\right)$ expected amortized time:

- INSERT $(u, v)$ : Insert the edge $(u, v)$ with resistance 1 in $G$.

- Delete $(u, v)$ : Delete the edge $(u, v)$ from $G$.

- ChANGe $\left(u, \mathbf{b}^{\prime}(u), v, \mathbf{b}^{\prime}(v)\right)$ : Change $\mathbf{b}(u)$ to $\mathbf{b}^{\prime}(u)$ and $\mathbf{b}(v)$ to $\mathbf{b}^{\prime}(v)$ while keeping $\mathbf{b}$ in the range of $\mathbf{L}$.

- $\operatorname{SOLVE}(u)$ :Return $\tilde{\mathbf{x}}(u)$ with $\tilde{\mathbf{x}}$ such that $\left\|\tilde{\mathbf{x}}-\mathbf{L}^{\dagger} \mathbf{b}\right\|_{\mathbf{L}} \leq \epsilon\left\|\mathbf{L}^{\dagger} \mathbf{b}\right\|_{\mathbf{L}}$.

Our guarantees hold against an oblivious adversary.

Note that the $\tilde{\mathbf{x}}$ in the theorem above is not guaranteed to be inside the range of $\mathbf{L}_{G}$ and it only preserves the differences between vertices in the same connected component.

We observe that conditioning on the vector $\mathbf{b}$ having small support, i.e., a small number of non-zero elements, immediately leads to a dynamic solver by just including the corresponding vertices into the Schur complement, and maintaining a dynamic Schur complement onto these vertices augmented with some carefully chosen additional terminals. Upon receipt of a query index, we add the corresponding vertex to the current Schur complement and simply solve a linear system there. However, note that the demand vector may have a large number of non-zero entries, thus preventing us from obtaining a sub-linear time algorithm with this approach. We alleviate this by projecting this vector onto the set of current 
terminals and showing that such projection can be maintained dynamically while introducing controllable error in the approximation guarantee.

A consequence of dynamic Laplacian solver is that we can maintain the energy of the electrical flow when routing any vector $b$ in the range of $\mathbf{L}$, which is a generalization of the All-Pairs Effective Resistance problem with $\mathbf{b}$ having exactly two non-zero entries, one of them being 1 and the other -1 . We defer such extension to the full version of the paper.

Another application of our technique is dynamic maintainance of effective resistance, a well studied quantity that has direct applications in random walks, spanning trees [44] and graph sparsification [60]. We maintain (approximate) All-Pairs Effective Resistances of a graph $G$ among any pair of query vertices while supporting an intermixed sequence of edge insertions and deletions in $G$. Our study is also motivated in part by the wide usage of commute distances, a random walk-based similarity measure that has been successfully employed in important practical applications such as link predictions [40]. Since commute distance is a scaled version of effective resistance, our dynamic algorithm readily extends to this graph measure while achieving the same approximation and running time guarantees.

THEOREM 1.3. For any given error threshold $\epsilon>0$, there is a datastructure for maintaining an unweighted, undirected multi-graph $G=(V, E)$ with up to $m$ edges that supports the following operations in $\tilde{O}\left(\mathrm{~m}^{3 / 4} \epsilon^{-4}\right)$ expected amortized time:

- INSERT $(u, v)$ : Insert the edge $(u, v)$ with resistance 1 in $G$.

- DeLETE $(u, v)$ : Delete the edge $(u, v)$ from $G$.

- EFFECTIVERESISTANCE $(s, t)$ : Return a $(1 \pm \epsilon)$-approximation to the effective resistance between s and $t$ in the current graph $G$.

Our guarantees hold against an oblivious adversary.

Our algorithm can also handle weighted graphs, albeit with a bound of $\tilde{O}\left(\mathrm{~m}^{5 / 6} \epsilon^{-4}\right)$ on the expected amortized update and query time. By running this algorithm on the output of a dynamic spectral sparsifier [2], we obtain a bound of $\tilde{O}\left(n^{5 / 6} \epsilon^{-6}\right)$ per operation, which is truly sub-linear irrespective of graph density. We discuss such improvements in Sections 4.3.

We are optimistic that our algorithmic ideas could be useful for dynamically maintaining a wider range of graph properties. Both the results that we give dynamic algorithms for, vertex sparsifiers and Schur complements, have wide ranges of applications in static settings, with the latter being at the core of the 'Laplacian paradigm' of graph algorithms $[63,64]$. While it's less clear that solutions across multiple Laplacian solves can be propagated to each other as the input dynamically changes, repeated sparsification on the other hand represents a routine that composes and interacts well with a much wider range of primitives. As a result, we are optimistic that it can be used as a building block in dynamic versions of many existing applications of Laplacian solvers.

\subsection{Related Works}

The recent data structures for maintaining effective resistances in planar graphs $[22,23]$ drew direct connections between Schur complements and data structures for maintaining them in dynamic graphs. This connection is due to the preservation of effective resistances under vertex eliminations (Fact 2.2). From this perspective, the Schur complement can be viewed as a vertex sparsifier for preserving resistances among a set of terminal vertices.

The power of vertex or edge graph sparsifiers, which preserve certain properties while reducing problem sizes, has long been studied in data structures $[18,19]$. Ideas from these results are central to recent works on offline maintenance for 3-connectivity [52], generating random spanning trees [16], and new notions of centrality for networks [39]. Our result is the first to maintain such vertex sparsifiers, specifically Schur complements, for general graphs in online settings.

While the ultimate goal is to dynamically maintain (approximate) minimum cuts and maximum flows, effective resistances represent a natural 'first candidate' for this direction of work due to them having perfect vertex sparsifiers. That is, for any subset of terminals, there is a sparse graph on them that approximately preserves the effective resistances among all pairs of terminals. This is in contrast to distances, where it's not known whether such a graph can be made sparse, or in contrast to cuts, where the existence of such a dense graph is not known (assuming that we are not content with large constant or poly-logarithmic approximations).

Dynamic Graph Algorithms. The maintenance of graph properties in dynamic algorithms has been a major area of ongoing research in data structures. The problems being maintained include $2-$ or 3-connectivity [19, 30, 31], shortest paths [1, 10, 14, 27, 28], global minimum cut $[24,29,38,65]$, maximum matching [11, 26, 50], and maximal matching $[6,49,59]$. Perhaps most closely related to our work are dynamic algorithms that maintain properties related to paths [19, 21, 30, 32, 47, 48, 67]. In particular, the work of WulffNilsen [67] also utilizes the behavior of random walks under edge deletions to keep track of low-conductance cuts.

Dynamic algorithms for evaluating algebraic functions such as matrix determinant and matrix inverse has also been considered [55]. One application of such algorithms is that they can be used to dynamically maintain single-pair effective resistance. Specifically, using the dynamic matrix inversion algorithm, one can dynamically maintain exact $(s, t)$-effective resistance in $O\left(n^{1.575}\right)$ update time and $O\left(n^{0.575}\right)$ query time.

Vertex Sparsifiers. Vertex sparsifiers have been studied in more general settings for preserving cuts and flows among terminal vertices [12, 35, 46]. Efficient versions of such routines have direct applications in data structures, even when they only work in restricted settings: terminal sparsifiers on quasi-bipartite graphs [3] were core routines in the data structure for maintaining flows in bipartite undirected graphs [2].

Our data structure utilizes vertex sparsifiers, but in even more limited settings as we get to control the set of vertices to sparsify onto. Specifically, the local maintenance of this sparsifier under insertions and deletions hinges upon the choice of a random subset of terminals, while vertex sparsifiers usually need to work for any subset of terminals. Evidence from numerical algorithms [17, 36] suggest this choice can significantly simplify interactions between algorithmic components. We hope this flexibility can motivate further studies of vertex sparsifiers in more restrictive, but still algorithmically useful settings. 
Organization. The paper is organized as follows. We discuss preliminaries in Section 2 and give an overview of the key techniques in Section 3. After that we give a data-structure for dynamic Schur complement on unweighted graphs in Section 4, which can be applied to the dynamic All-Pairs Effective Resistance problem. In Section 4.3, we briefly discuss the extension of our data-structure to weighted graphs. In Section 5, we give a high-level idea behind a data-structure that dynamically maintains the projection of a vector onto a subset of vertices of an unweighted bounded degree graph, which can be combined with dynamic Schur complement to give a dynamic Laplacian solver. All missing algorithms and proofs are deferred to the full version of the paper.

\section{PRELIMINARIES}

In our dynamic setting, an undirected, weighted multi-graph undergoes both insertions and deletions of edges. We let $G=(V, E, \boldsymbol{w})$ always refer to the current version of the graph. We will use $n$ and $m$ to denote bounds on the number of vertices and edges at any point, respectively.

For an unweighted, undirected multi-graph $G$, let $\mathbf{A}_{G}$ denote its adjacency matrix and let $\mathbf{D}_{G}$ its degree diagonal matrix (counting edge multiplicities for both matrices). The graph Laplacian $\mathbf{L}_{G}$ of $G$ is then defined as $\mathbf{L}_{G}=\mathbf{D}_{G}-\mathbf{A}_{G}$. Let $\mathbf{L}_{G}^{\dagger}$ denote the Moore-Penrose pseudo-inverse of $\mathbf{L}_{G}$. Subscripts will be often omitted when the underlying graph is clear from the context. We define the indicator vector $1_{u} \in \mathbb{R}^{n}$ of a vertex $u$ such that $1_{u}(v)=1$ if $v=u$, and $\mathbf{1}_{u}(v)=0$ otherwise.

A walk in $G$ is a sequence of vertices such that consecutive vertices are connected by edges. A random walk in $G$ is a walk that starts at a starting vertex $w_{0}$, and at step $i \geq 1$, the vertex $w_{i}$ is chosen randomly among the neighbors of $w_{i-1}$. If graph $G$ is unweighted, then each of its neighbors becomes $w_{i}$ with equal probability. If $G$ is weighted, the probability $\mathbb{P}_{w}\left[w_{i}=u \mid w_{0}, \ldots, w_{i-1}\right]$ is proportional to the edge weight $\boldsymbol{w}_{w_{i-1} u}$.

Effective Resistance. For our algorithm, it will be useful to define effective resistance using linear algebraic structures. Specifically, given any two vertices $u$ and $v$ in $G$, if $\chi(u, v):=\mathbf{1}_{u}-\mathbf{1}_{v}$, then the effective resistance between $u$ and $v$ is given by

$$
R_{\mathrm{eff}}^{G}(u, v):=\chi_{u, v}^{\top} \mathbf{L}_{G}^{\dagger} \chi_{u, v} .
$$

Linear systems in graph Laplacian matrices can be solved in nearly-linear time [34]. One prominent application of these solvers is the approximation of effective resistances.

Lemma 2.1. Fix $\epsilon \in(0,1)$ and let $G=(V, E)$ be any graph with two arbitrary distinguished vertices $u$ and $v$. There is an algorithm that computes a value $\phi$ such that

$$
(1-\epsilon) R_{\mathrm{eff}}^{G}(u, v) \leq \phi \leq(1+\epsilon) R_{\mathrm{eff}}^{G}(u, v),
$$

in $\tilde{O}\left(m+n / \epsilon^{2}\right)$ time with high probability.

Schur complement. Given a graph $G=(V, E)$, we can think of the Schur complement as the partially eliminated state of $G$. This relies on some partitioning of $V$ into two disjoint subset of vertices $T$, called terminals and $F:=V \backslash T$, called non-terminals, which in turn partition the Laplacian $\mathrm{L}$ into 4 blocks:

$$
\mathbf{L}:=\left[\begin{array}{ll}
\mathbf{L}_{[F, F]} & \mathbf{L}_{[F, T]} \\
\mathbf{L}_{[T, F]} & \mathbf{L}_{[T, T]}
\end{array}\right] .
$$

The Schur complement onto $T$, denoted by $\operatorname{SC}(G, T)$, is the matrix obtained after eliminating the variables in $F$. Its closed form is given by

$$
\operatorname{SC}(G, T)=\mathbf{L}_{[T, T]}-\mathbf{L}_{[T, F]} \mathbf{L}_{[F, F]}^{-1} \mathbf{L}_{[F, T]}
$$

It is well known that $\operatorname{SC}(G, T)$ is a Laplacian matrix of a graph on vertices in $T$. To simplify our exposition, we let $\operatorname{SC}(G, T)$ denote both the Laplacian and its corresponding graph. An important property of Schur complement which we exploit in this work is to view the Schur complement as a collection of random walks. This particular feature will be discussed in more detail in Section 3. The key role of Schur complements in our algorithms stems from the fact that they can be viewed as vertex sparsifiers that preserve pairwise effective resistances (see e.g., [22]).

Fact 2.2 (Vertex Resistance Sparsifier). For any graph $G=$ $(V, E)$, any subset of vertices $T$, and any pair of vertices $u, v \in T$,

$$
R_{\mathrm{eff}}^{G}(u, v)=R_{\mathrm{eff}}^{\mathrm{SC}(G, T)}(u, v) .
$$

\section{Spectral Approximation.}

Definition 2.3 (Spectral Sparsifier). Given a graph $G=(V, E)$ and $\epsilon \in(0,1)$, we say that a graph $H=\left(V, E^{\prime}\right)$ is a $(1 \pm \epsilon)$-spectral sparsifier of $G$ (abbr. $H \approx_{\epsilon} G$ ) if $E^{\prime} \subseteq E$, and for all $\mathbf{x} \in \mathbb{R}^{n}$

$$
(1-\varepsilon) \mathbf{x}^{\top} \mathbf{L}_{G} \mathbf{x} \leq \mathbf{x}^{\top} \mathbf{L}_{H} \mathbf{x} \leq(1+\varepsilon) \mathbf{x}^{\top} \mathbf{L}_{G} \mathbf{x} .
$$

In the dynamic setting, Abraham et al. [2] recently showed that $(1 \pm \epsilon)$-spectral sparsifiers of a dynamic graph $G$ can be maintained efficiently. This algorithm will be invoked in several places throughout this paper.

Lemma 2.4 ([2], Theorem 4.1). Given a graph $G$ with polynomially bounded edge weights, with high probability, we can dynamically maintain a $(1 \pm \epsilon)$-spectral sparsifier of size $\tilde{O}\left(n \epsilon^{-2}\right)$ of $G$ in $O\left(\log ^{9} n \epsilon^{-2}\right)$ expected amortized time per edge insertion or deletion. The running time guarantees hold against an oblivious adversary.

The above result is useful because matrix approximations also preserve approximations of their quadratic forms. As a consequence of this fact, we get the following lemma.

LEMMA 2.5. If $H$ is a $(1 \pm \epsilon)$-spectral sparsifier of $G$, then for any pair of vertices $u$ and $v$

$$
(1-\varepsilon) R_{\mathrm{eff}}^{G}(u, v) \leq R_{\mathrm{eff}}^{H}(u, v) \leq(1+\varepsilon) R_{\mathrm{eff}}^{G}(u, v) .
$$

\subsection{Projection matrix and its properties}

We next define a matrix that naturally appears when performing Gaussian elimination on the non-terminal vertices. Concretely, given a graph $G=(V, E)$ and terminals $T \subseteq V$, the matrix-projection of the non-terminals $F=V \backslash T$ onto $T$ is given by

$$
\mathbf{P}(T):=\left[\begin{array}{ll}
-\mathbf{L}_{[T, F]} \mathbf{L}_{[F, F]}^{-1} & \mathbf{I}_{T}
\end{array}\right] .
$$

We now review some useful properties about the matrix projection $\mathbf{P}(T)$. Consider the Laplacian system $\mathbf{L x}=\mathbf{b}$, where $\mathbf{L}$ is partitioned into block-matrices as in Equation (1). This in turn partitions the solution vector into non-terminals and terminals, i.e., $\mathbf{x}=\left[\mathbf{x}_{F} \mathbf{x}_{T}\right]^{\top}$. 
LEMMA 2.6. Let $\mathbf{x}_{T}$ be a solution vector such that $\operatorname{SC}(G, T) \mathbf{x}_{T}=$ $\mathbf{P}(T) \mathbf{b}$. Then there exists an extension $\mathbf{x}$ of $\mathbf{x}_{T}$ such that $\mathbf{L x}=\mathbf{b}$.

The following lemma draws a connection between the projection matrix and certain random walk probabilities which will allow us to take a combinatorial view on several problems we deal with.

Lemma 2.7. Consider a graph $G=(V, E)$. For any subset of vertices $T \subseteq V$, a vertex $v \in T$, and a vertex $u \in F=V \backslash T$, let $\mathbb{P}_{u}\left[t_{v}<t_{T \backslash v}\right]$ be the probability that the random walk that starts at $u$ hits $v$ before hitting any other vertex from $T \backslash v$. Then we have that

$$
\left[\mathbf{P}(T) 1_{u}\right](v)=\mathbb{P}_{u}\left[t_{v}<t_{T \backslash v}\right] .
$$

In fact, $\left\{\mathbf{P}(T) \mathbf{1}_{u}\right\}_{v \in T}$ is a probability distribution for any fixed vertex $v \in F$.

Given a demand vector $\mathbf{b} \in \mathbb{R}^{n}$, we say that $\mathbf{P}(T) \cdot \mathbf{b}$ is the projection of $\mathbf{b}$ onto $T$. In general, the projection of $\mathbf{b}$ is shorter than the original vector $\mathbf{b}$. However, for the sake of exposition, often we consider $\mathbf{P}(T) \cdot \mathbf{b}$ to be an $n$-dimensional vector by assuming that all coordinates in $F$ are 0 .

Lemma 2.8. Consider a graph $G=(V, E)$. Let $T \subseteq V$ be a subset of vertices, and let $u \in F=V \backslash T$. Consider the demand vector $\mathbf{1}_{u}-$ $\mathrm{P}(T) 1_{u}$ that requests to send one unit of flow from $u$ to $T$ according to the probability distribution $\left\{\mathbf{P}(T) \mathbf{1}_{u}\right\}_{v \in T}$. Then the minimum energy needed to route this demand is given by

$$
\left\|\mathbf{1}_{u}-\mathbf{P}(T) \mathbf{1}_{u}\right\|_{\mathbf{L}^{\dagger}}^{2}=\left(\mathbf{1}_{u}-\mathbf{P}(T) \mathbf{1}_{u}\right)^{\top} \mathbf{L}^{\dagger}\left(\mathbf{1}_{u}-\mathbf{P}(T) \mathbf{1}_{u}\right) .
$$

\section{OVERVIEW}

The core building block of our algorithms is a fast routine that generates and maintains an approximate Schur complement onto a set of terminals $T$ of our choice under insertion and deletions of edges as well as terminal additions, with all of these operations being supported in sub-linear time. One of the key ideas is to view to the Schur complement as a sum of random walks, and then observe that sampling exactly one walk per edge in the original graph already yields the desired object. Concretely, we build upon ideas introduced in sparsifying random walk polynomials [13], and Schur complements $[17,36]$ to show that it suffices to keep a union of these walks. The following result is implicit in these works, and we will review its proof in the full version of this paper.

Theorem 3.1. Let $G=(V, E, w)$ be an undirected, weighted multigraph with a subset of vertices $T$. Furthermore, let $\epsilon \in(0,1)$, and let $\rho$ be some parameter related to the concentration of sampling given by

$$
\rho=O\left(\log n \epsilon^{-2}\right) .
$$

Let $H$ be an initially empty graph, and for every edge $e=(u, v)$ of repeat $\rho$ times the following procedure:

(1) Simulate a random walk starting from $u$ until it first hits $T$ at vertex $t_{1}$,

(2) Simulate a random walk starting from $v$ until it first hits $T$ at vertex $t_{2}$,

(3) Combine these two walks (including $e)$ to get a walk $u=\left(t_{1}=\right.$ $\left.u_{0}, \ldots, u_{\ell}=t_{2}\right)$, where $\ell$ is the length of the combined walk.
(4) Add the edge $\left(t_{1}, t_{2}\right)$ to $H$ with weight

$$
1 /\left(\rho \sum_{i=0}^{\ell-1}\left(1 / w_{u_{i} u_{i+1}}\right)\right)
$$

The resulting graph $H$ satisfies $\mathbf{L}_{H} \approx_{\epsilon} \mathrm{SC}(G, T)$ with high probability.

The output approximate Schur complement of $H$ onto $T$ has up to $\tilde{O}\left(m \epsilon^{-2}\right)$ edges, and thus is very dense to be leveraged as a sparsifier for our applications. Fortunately, there already exist efficient dynamic spectral sparsifiers, and we can always afford to keep a sparsifier $\tilde{H}$ of $H$ whose size is only $\tilde{O}\left(|T| \epsilon^{-2}\right)$.

The performance of our data structure depends on how fast we can generate the random walks used to create $H$. Note that even on the length $n$ path with terminals $T$ concentrated on one end, the lengths of these walks may be as long as $\Omega\left(n^{2}\right)$. To overcome this we shorten the walks by augmenting $T$ with roughly $O(\beta m)$ random vertices from a carefully chosen distribution. This random augmentation of $T$ ensures that any vertex $v$ in $G$ is roughly $O\left(\beta^{-1}\right)$ apart from a vertex in $T$, and then our problem reduces to understanding the rate at which a random walk spreads among distinct edges. Concretely, our goal is to efficiently generate the first $k$ distinct edges visited by a walk in $G$. We distinguish the following cases.

(1) For unweighted graphs, we utilize a result by Barnes and Feige [5] that shows that with high probability a walk reaches $k$ distinct edges in about $k^{2}$ steps.

(2) For weighted graphs, we employ an event driven simulation of walks. Specifically, by computing the exit probability on the current set of edges visited so far, we sample the first $k$ new edges reached by the walk in poly $(k)$ time. Then, because we know the order that each edges is first reached, the first among them that belongs to $T$ gives the intersection of the walk with $T$.

Following Point (1), our dynamic Schur complement datastructure $H$ with respect to a randomly augmented $T$ is initialized by generating for each edge $e \in E, \rho$ random walk of length roughly $\beta^{-2}$. This operation costs roughly $O\left(m \beta^{-2}\right)$. We then make the observation that the ability to add terminals into $T$ means we only need to consider insertions/deletions between vertices in $T$. Specifically, for each affected edge we append its endpoints to $T$. A further advantage of this approach is that additions to $T$ only shorten random walks in $H$, and the cost of shortening or truncating these random walks in $H$ can be charged to the cost of constructing them during the initialization. Thus, it follows that we can support terminal additions, and thus insert or delete edges in $O(1)$ amortized time. Maintaining a sparsifier $\tilde{H}$ of $H$ introduces only polylogarithmic overheads, so this step does not affect much our running times. We next discuss the applicability of this result.

The data-structure we presented readily gives a sub-linear dynamic Laplacian solver for the case where $\mathbf{b}$ has small support, namely fewer than $\beta m$ vertices of $\mathbf{b}$ are non-zero. This can be accomplished by simply appending the entries of $\mathbf{b}$ (more precisely, their corresponding vertices) to the Schur complement $H$, and solving the system on $H$ upon receipt of an index query. The resulting solution vector can then be lifted back to the original Laplacian using Lemma 2.6. However, note that our data-structure can only 
support up to $O(|T|)=O(\beta m)$ operations if we want to keep the the size of $H$ small. Thus, to limit the growth in $|T|$ we periodically rebuild the entire data structure (i.e., we resample the set of new terminals completely) after $\beta m$ operations, which in turn gives an amortized update time of $O\left(m \beta^{-2} /(\beta m)\right)=O\left(\beta^{-3}\right)$. Combining this with the bound of $O(\beta m)$ on the query time we obtain the following trade-off

$$
\tilde{O}\left(\beta^{-3}+\beta m\right)
$$

which is minimized when $\beta=m^{-1 / 4}$, thus giving an update and query time of $O\left(\mathrm{~m}^{3 / 4}\right)$.

So it remains to address the case where $b$ has a large number of non-zero entries. We overcome this difficulty by projecting this vector onto the current set of terminals $T$ using the matrix $\mathbf{P}(T)$ and analyzing the error incurred by this projection. Our main observation is that the standard notion of error in Laplacian solvers, namely the L-norm, corresponds to energies of electrical flows. This allows us to incur error in some of the $\mathbf{b}(u)$ values and then bound the energy of fixing them. To find such flows, we once again consider our problem from a random walk perspective, namely we view the projection of $\mathbf{b}$ onto $T$ being equivalent to moving $\mathbf{b}$ around via random walks (Lemma 2.7). As such walks are short on unweighted graphs, we can relate their energies to the length of the walks times b $(u)^{2}$ (Lemma 2.8).

One final obstacle is that if we move some vertex $u$ from outside of $T$ into $T$, the walks affected may be from multiple $\mathbf{b}(u)$ s. To address this, we bound the 'load' of a vertex, defined as the number of walks that go through it, by the total length of the walks. The latter follows from the uniform distribution of random walks being stationary. Thus, as long as we picked $T$ so that all the entries in $V \backslash T$ have small magnitudes, each move of some $u$ into $T$ incurs some small error. Bounding the accumulation of such errors, and rebuilding appropriately gives the overall dynamic solver result.

One application of the dynamic Laplacian solver is that we can maintain the energy of electrical flow for routing $\mathbf{b}$. This can also be viewed as an extension of our dynamic effective resistances data-structure, which can only maintain the energy of electrical flows for $\mathbf{b}$ with two non-zeros. Some further extensions in this direction that we believe would be useful are providing implicit access to the dual electrical flows, as well as finding the $k$ largest entries either in the flow edges or the solution vector $\mathbf{x}$. However, such extensions will likely require a better understanding of the graph sparsifier component [2], which is treated as a black box in this paper.

For dynamically maintaining effective resistance in unweighted graphs, we essentially follow the same approach as with the dynamic solver for small support demand vectors, and thus obtain a running time of $O\left(\mathrm{~m}^{3 / 4}\right)$ on both update and query time. For weighted graphs, we employ the weighted dynamic Schur complement algorithm (following Point(2)) which gives slightly weaker guarantees, namely a bound of $\tilde{O}\left(\mathrm{~m}^{5 / 6}\right)$ on the update and query time. Interestingly, this weighted version has another immediate advantage; by running the data-structure on the output of a dynamic spectral sparsifier (Lemma 2.4), we obtained a bound of $\tilde{O}\left(n^{5 / 6}\right)$ per operation, which is truly sub-linear irrespective of graph density.

\section{DYNAMIC SCHUR COMPLEMENT}

In this section we show how to dynamically maintain approximate Schur complements. We first restrict our attention to unweighted graphs (i.e., prove Lemma 1.1), and then discuss how this result extends to the weighted case. We also present one of the applications of our data-structure, namely dynamic maintenance of effective resistance on unweighted (Theorem 1.3).

\subsection{Dynamic Schur Complement on Unweighted Graphs}

In this section we design a data-structure for maintaining approximte Schur complements under the assumption that the dynamic graph remains unweighted throughout the updates. Specifically, we have the following lemma.

Lemma 4.1 (Restatement of Lemma 1.1). Given an error threshold $\epsilon>0$, an unweighted undirected multi-graph $G=(V, E)$ with $n$ vertices, $m$ edges, a subset of terminal vertices $T^{\prime}$ and a parameter $\beta \in(0,1)$ such that $\left|T^{\prime}\right|=O(\beta m)$, there is a datastructure DYNAMICSC $\left(G, T^{\prime}, \beta\right)$ for maintaining a graph $\tilde{H}$ with $\mathrm{L}_{\tilde{H}} \approx_{\epsilon} \operatorname{SC}(G, T)$ for some $T$ with $T^{\prime} \subseteq T,|T|=O(\beta m)$, while supporting $O(\beta m)$ operations in the following running times:

- Initialize $\left(G, T^{\prime}, \beta\right)$ : Initialize the data-structure, in $\tilde{O}\left(m \beta^{-2} \epsilon^{-4}\right)$ expected amortized time.

- INSERT $(u, v)$ : Insert the edge $(u, v)$ to $G$ in Õ $(1)$ amort. time.

- DeLETE $(u, v)$ : Delete the existing edge $(u, v)$ from $G$ in Õ $(1)$ amortized time.

- AdDTerminal (u): Add u to $T^{\prime}$ in Õ(1) amortized time.

Our guarantees hold against an oblivious adversary.

To prove the lemma above, we first review the interpretation of Schur Complements using random walks, and then discuss how to generate and maintain these walks under edge updates and addition of terminal vertices.

Given a graph $G=(V, E)$ and a subset of terminals $T$ recall that $\operatorname{SC}(G, T)$ was defined using an algebraic expression that involved the Laplcian of $G$. However, since it is still unclear how to exploit this expression in the dynamic setting we instead take a different, more 'combinatorial', view on $\operatorname{SC}(G, T)$. Concretely, we will interpret $\operatorname{SC}(G, T)$ as a collection of random walks, each starting at an edge of $G$ and terminating in $T$, as described in Theorem 3.1.

Let $H$ be the output graph from the construction in Theorem 3.1. Recall that $H$ is an approximate Schur Complement onto $T$ that has up to $\rho m=\tilde{O}\left(m \epsilon^{-2}\right)$ edges (that is, $\rho$ for each edge in $G$, where $\rho=O\left(\log n \epsilon^{-2}\right)$ is the sampling parameter). As we will next show, $H$ does not change too much (in amortized sense) upon inserting or deleting an edge in $G$. We will be able to maintain $H$ such that the distribution of $H$ is the same as $H(G)$ of the current graph $G$. Therefore, we can maintain these changes using a dynamic spectral sparsifier $\tilde{H}$ of $H$ (Lemma 2.4), and whenever a query comes, we answer it on $\tilde{H}$ in $\tilde{O}\left(|T| \epsilon^{-2}\right)=\tilde{O}\left(\beta m \epsilon^{-2}\right)$ time.

While it is widely known how to generate random walks efficiently, we note that the length of the walks generated in Theorem 3.1 could be prohibitively large if $T$ is being picked arbitrarily. To see this, recall our example where we considered a path of length $n$ with terminals $T$ being places in one end. The length of such random walks may be as long as $\Omega\left(n^{2}\right)$. To shorten these random walks, 
we augment $T$ with a random subset of vertices. Coming back to the path example, $\beta n$ uniformly random vertices will be roughly $\beta^{-1}$ apart, and random walks will reach one of these $\beta n$ vertices in about $\beta^{-2}$ steps. Because $G$ could be a multi-graph, and we want to support queries involving any vertex, we pick $T$ as the end points of a uniform subset of edges. A case that illustrates the necessity of this choice is a path except one edge has $n$ parallel edges. In this case it takes $\Theta(n)$ steps in expectation for a random walk to move away from the end points of that edge. This choice of $T$ completes the definition of our data structure, which we summarize in Algorithm 1. The performance of our data structures hinge upon the properties

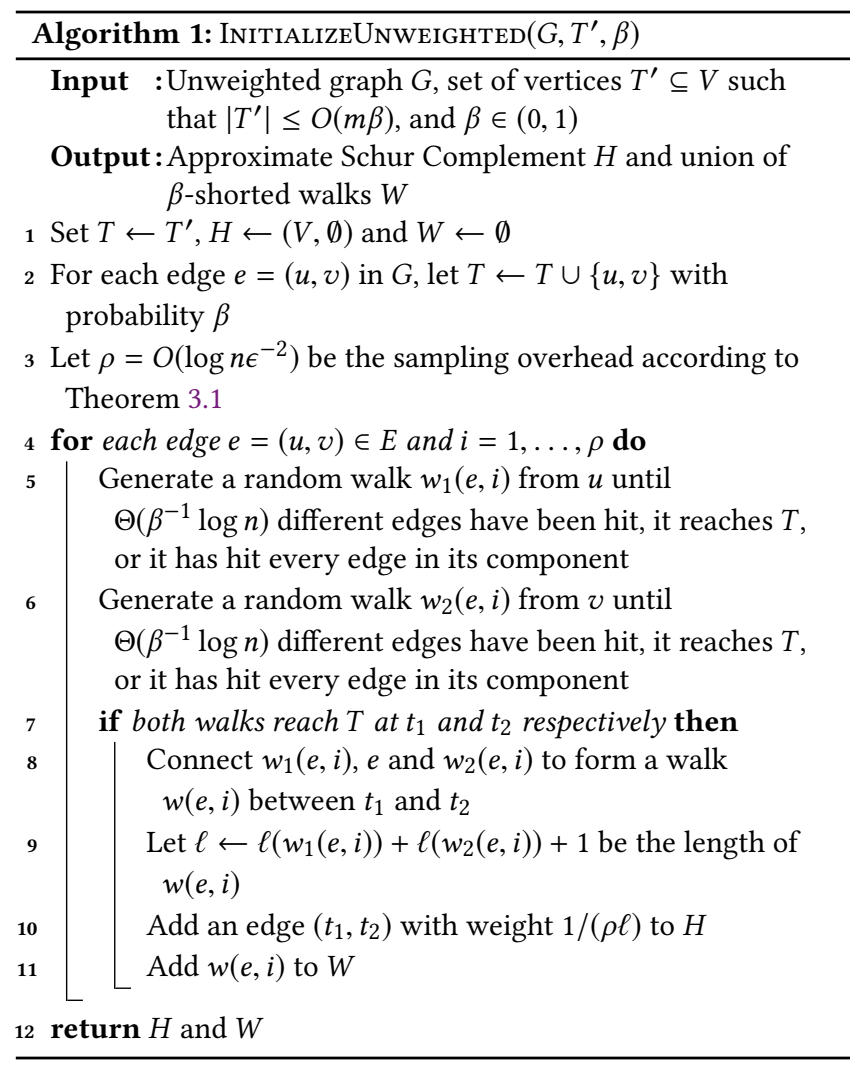

of the generated random walks. We start by formalizing such a structure involving the set of the augmented terminals described above while parameterizing it with a more general probability $\beta$ for including the endpoints of the edges.

Definition 4.2 ( $\beta$-shorted walks). Let $G$ be an weighted, undirected multi-graph and $\beta \in(0,1)$ a parameter. A collection of $\beta$-shorted walks $W$ on $G$ is a set of random walks created as follows:

(1) Choose a subset of terminal vertices $T$, obtained by including the endpoints of each edge independently with probability at least $\beta$.

(2) For each edge $e \in E$, generate $\rho=O\left(\log n \epsilon^{-2}\right)$ walks from its endpoints either until $\Omega\left(\beta^{-1} \log n\right)$ different edges have been hit, they reach $T$, or they visited each edge that is in the same connected component as $e$.
As we will shortly seee, the main property of the collection $W$ is that its random walks are short. Moreover, we will also prove that all walks in $W$ will reach $T$ with high probability. These guarantees are summarized in the following theorem.

Theorem 4.3. Let $G=(V, E)$ be any undirected multi-graph, and $\beta \in(0,1)$ a parameter. Any set of $\beta$-shorted walks $W$, as described in Definition 4.2, satisfies the following:

- With high probability, any random walk in $W$ starting in a connected component containing a vertex from $T$ terminates at a vertex in $T$.

Note that Theorem 4.3 is conditioned upon the connected component having a vertex in $T$ : this is necessary because walks stay inside a connected component. However, this does not affect our queries: our data-structure has an operation for making any vertex $u$ a terminal, which we call during each query to ensure both $s$ and $t$ are terminal vertices. Such an operation interacts well with Theorem 4.3 because it can only increase the probability of an edge's endpoints being chosen.

Proving the theorem requires to determine the rate at which a random walk visits at least $\beta^{-1} \log n$ edges. It turns out that a random walk of length $\tilde{O}\left(\beta^{-2}\right)$ is highly likely to achieve this. For formally showing this, we need the following result by Barnes and Feige [5].

Theorem 4.4 ([5], Theorem 1.2). There is an absolute constant $c_{B F}$ such that for any undirected unweighted connected multi-graph $G$ with $n$ vertices and $m$ edges, any vertex $u$ and any value $\hat{m} \leq m$, the expected time for a random walk starting from $u$ to visit at least $\hat{m}$ distinct edges is at most $c_{B F} \hat{m}^{2}$.

The above theorem can be amplified into a with high probability bound by repeating the walk $O(\log n)$ times.

COROLlary 4.5. In any undirected unweighted connected multigraph $G$ with $m$ edges, for any starting vertex $u$, any length $\ell \leq m^{2}$, and a parameter $\delta \geq 1$, a walk of length $c_{B F} \cdot \delta \cdot \ell \log n$ from $u$ visits at least $\ell^{1 / 2}$ distinct edges with probability at least $1-n^{-\delta}$.

Proof. We can view each such walk as a concatenation of $\delta \log n$ sub-walks, each of length $2 \cdot c_{B F} \cdot \ell$.

We call a sub-walk good if the number of distinct edges that it visits is at least $\ell^{1 / 2}$. Applying Markov's inequality to the result of Theorem 4.4, a walk takes more than $2 \cdot c_{B F} \cdot \ell$ steps to visit $\ell^{1 / 2}$ distinct edges with probability at most $1 / 2$.

This means that each subwalk fails to be good with probability at most $1 / 2$. Thus, the probability that all subwalks fail to be good is at most $2^{-\delta \log n}=n^{-\delta}$. The result then follows from an union bound over all starting vertices $u \in V$.

We now have all the tools to prove Theorem 4.3.

Proof of Theorem 4.3. For any walk $w$, we define $V(w)$ (respectively, $E(w)$ ) to be the set of distinct vertices (respectively, edges) that a walk $w$ visits. Consider a random walk $w$ that starts at $u$ of length

$$
\ell=c_{B F} \cdot \delta^{3} \cdot \beta^{-2} \log ^{3} n
$$

where $\delta$ is a constant related to the success probability. 
If the connected component containing the walk has fewer than

$$
\delta \cdot \beta^{-1} \cdot \log n
$$

edges, then Corollary 4.5 gives that we have covered this entire component with high probability, and the guarantee follows from the assumption that this component contains a vertex of $T$.

Otherwise, we will show that $w$ reached enough edges for one of their endpoints to be picked to be picked into $T$ with high probability. The key observation is that because $w$ is generated independently from $T$, we can bound the probability of this walk not hitting $T$ by first generating $w$, and then $T$. Specifically, for any size threshold $z$, we have

$$
\begin{aligned}
\mathbb{P}_{T, w}[V(w) \cap T=\emptyset] & =\mathbb{P}_{w, T}[V(w) \cap T=\emptyset] \\
\leq & \mathbb{P}_{w}[|E(w)| \leq z] \\
& +\mathbb{P}_{w:|E(w)| \geq z, T}[V(w) \cap T=\emptyset] .
\end{aligned}
$$

By Corollary 4.5 and the choice of $\ell$, if we set

$$
z=\delta \cdot \beta^{-1} \cdot \log n,
$$

then the first term in Equation (2) is bounded by $n^{-\delta}$. For bounding the second term, we can now focus on a particular walk $\widehat{w}$ that visits at least $\delta \cdot \beta^{-1} \cdot \log n$ distinct edges, i.e.,

$$
|E(\widehat{w})| \geq \delta \cdot \beta^{-1} \log n .
$$

Recall that we independently added the end points of each of these edges into $T$ with probability $\beta$. If any of them is selected, we have a vertex that is both in $V(\widehat{w})$ and $T$. Thus, the probability that $T$ contains no vertices from $V(\widehat{w})$ is at most

$$
(1-\beta)^{|E(\widehat{w})|} \leq(1-\beta)^{\delta \cdot \beta^{-1} \log n} \leq e^{-\delta \log n} \leq n^{-\delta},
$$

which completes the proof.

Corollary 4.5 together with Theorem 4.3 yield the following lemma.

Lemma 4.6. Algorithm 1 runs in $\tilde{O}\left(m \beta^{-2} \epsilon^{-2}\right)$ time and outputs a graph $H$ with $\mathrm{L}_{H} \approx_{\epsilon} \mathrm{SC}(G, T)$, with high probability.

Proof. By Corollary 4.5, the length of each walk generated in Algorithm 1 is bounded by $O\left(\beta^{-2} \log ^{3} n\right)$. In addition, note that each step in a random walk can be simulated in $O(1)$ time. This is due to the fact that we can sample an integer in $[0, n-1]$ by drawing $x \in[0,1]$ uniformly and taking $\lfloor x n\rfloor$. Combining these with the fact that the algorithm generates $\rho m=\tilde{O}\left(m \epsilon^{-2}\right)$ walks, it follows that the running time of the algorithm is dominated by $\tilde{O}\left(m \beta^{-2} \epsilon^{-2}\right)$.

Note that the collection of generated walks form the set $W$ of $\beta$-shorted walks. By Theorem 4.3, with high probability, each of the walks that starts at a component containing a vertex in $T$ hits $T$. Conditioning on the latter, Theorem 3.1 gives that with high probability, $\mathbf{L}_{H} \approx \epsilon \operatorname{SC}(G, T)$.

Handling edge updates and terminal additions. We start by observing that there is always a one-to-one correspondence between the collection of $\beta$-shorted walks $W$ and our approximate Schur complement $H$. Accordingly, our primary concern will be supporting the InSERT, Delete, and AddTerminal operations in the collection $W$. However, as $W$ undergoes changes, we need to efficiently update the sparsifier $H$. To handle these updates, we would ideally have efficient access to walks in $W$ that are affected by the corresponding updates.

To achieve this, we index into walks that utilize a vertex or an edge, and thus set up a reverse data structure pointing from vertices and edges to the walks that contain them. The following lemma says that we can modify this representation with minimal cost.

Lemma 4.7. For the collection of $\beta$-shorted walks $W$, let $W_{v}$ and $W_{e}$ be the specific walks of $W$ that contain vertex $v$ and edge e, respectively. We can maintain a data structure for $W$ such that for any vertex $v$ or edge e it reports

- all walks in $W_{v}$ or $W_{e}$ in $O\left(\left|W_{v}\right|\right)$ or $O\left(\left|W_{e}\right|\right)$ time, respectively, with an additional $O(\log n)$ overhead for any changes made to $W$.

Proof. For every vertex (respectively, edge), we can maintain a balanced binary search tree consisting of all the walks that use it in time proportional to the number of vertices (respectively, edges) in the walks. Supporting rank and select operations on such trees then gives the claimed bound.

As a result, any update made to the collection of walks can be updated in the approximate Schur complement $H$ generated from these walks in $O(\log n)$ time. We now have all the necessary ingredients to prove Lemma 1.1.

\begin{tabular}{l}
\hline Algorithm 2: AddTerminal $(u)$ \\
\hline Input $:$ Vertex $u$ such that $u \notin T$ \\
1 Set $T \leftarrow T \cup\{u\}$ \\
2 Shorten all random walks in $W$ at the first point they meet $u$ \\
3 Update the corresponding edges in $H$ and $\tilde{H}$ \\
\hline
\end{tabular}

Proof of Lemma 1.1. We give a two-level data-structure for dynamically maintaining Schur complements on unweighted graphs, which keeps at any time a terminal set $T$ of size $\Theta(m \beta)$. This entails maintaining

(1) an approximate Schur complement $H$ of $G$ with respect to $T$ (Theorem 3.1),

(2) a dynamic spectral sparsifier $\tilde{H}$ of $H$ (Lemma 2.4).

We implement the procedure INITIALIzE by running Algorithm 1, which produces a graph $H$ and then computing a spectral sparsifier $\tilde{H}$ of $H$ using Lemma 2.4. Note that by construction of our data-structure, every update in $H$ will be handled by the black-box dynamic sparsifier $\tilde{H}$.

As we will shortly see, operations InSERT and Delete will be reduced to adding terminals to the set $T$. Thus, the bulk of our effort is devoted to implementing the procedure ADDTERMINAL. Let $u$ be a non-terminal vertex that we want to append to $T$. We set $T \leftarrow T \cup\{u\}$, and then shorten all the walks at the first location they meet $u$. This shortening of walks induces in turn edge insertions and deletions to $H$, which are then processed by $\tilde{H}$. The pseudocode for this operation is summarized in Algorithm 2. To quickly locate the first appearances of $u$ in the random walks from $W$, we make use of the data-structure from Lemma 4.7. Let us first describe the construction of such data-structure during the preprocessing phase. Let $W_{u}$ be the balanced binary search tree consisting of all the 
walks that use the vertex $u$ in $W$. Fix $w \in W_{u}$. For any $t \geq 0$, if $w$ visits $u$ after $t$ steps, we check whether $W_{u}$ contains $w$ or not. If the latter holds, we know that $u$ has appeared before in $w$ and we do not need to add $w$ to $W_{u}$. Otherwise, we add $w$ to $W_{u}$ as this is the first time the walk $w$ visits $u$. After locating the first appearances of $u$, we cut the walks in these locations, delete the corresponding affected walks (together with their weight from $H$ ), and insert the new shorter walks to $H$. Note that we can simply use arrays to represent each random walk in $W$.

For implementing operations INSERT and DELETE we proceed as follows. Specifically, upon insertion or deletion of an edge $e=(u, v)$ in $G$, we append both $u$ and $v$ to the terminal set $T$. Now, all the walks that pass through $u$ or $v$ in $W$ must be shorten at the first location they meet $u$ or $v$. For inserting an edge $(u, v)$ with weight $\boldsymbol{w}_{u v}$ in $G$ (in fact, Lemma 1.1 is restricted to $\boldsymbol{w}_{u, v}=1$ ), we simply add $\rho$ trivial random walks (i.e., the edge $(u, v))$ of weight $\frac{w_{u v}}{\rho}$ to $H$ (which sum up to the edge $(u, v)$ itself). For deleting the edge $(u, v)$ with weight $\boldsymbol{w}_{u v}$ from $G$, simply delete these $\rho$ random walks between $u$ and $v$ in $H$ (which exist since we guaranteed that $u$ and $v$ are added as terminals to $H$ ).

We next analyze the performance of our data-structure. Let us start with the pre-processing time. First, by Lemma 4.6 we get that the cost for constructing $H$ on a graph with $m$ edges is bounded by $\tilde{O}\left(m \beta^{-2} \epsilon^{-2}\right)$. Next, since $H$ has $\tilde{O}\left(m \epsilon^{-2}\right)$ edges, constructing $\tilde{H}$ takes $\tilde{O}\left(m \epsilon^{-4}\right)$ time. Thus, the amortized time of InITIALIzE operation is bounded by $\tilde{O}\left(m \beta^{-2} \epsilon^{-4}\right)$.

Following the above discussion, for analyzing the cost of the update operations it suffices to bound the time for adding a vertex to $T$, which in turn (asymptotically) bounds the update time for edge insertions and deletions. The main observation we make is that adding a vertex to $T$ only shortens the existing walks, and Lemma 4.7 allows us to find such walks in time proportional to the amount of edges deleted from the walk. Since the walk needed to be generated in the INITIALIZE operation, the deletion of these edges takes equivalent time to generating them. Moreover, we note that (1) handling the updates in $\tilde{H}$ induced by $H$ introduces additional $O\left(\operatorname{poly}(\log n) \epsilon^{-2}\right)$ overheads, and (2) adding or deleting $\rho$ edges until the next rebuild costs $\tilde{O}\left(\beta m \epsilon^{-2}\right)$, since we process only up to $\beta m$ operations. These together imply that the amortized cost for adding a terminal can be charged against the pre-processing time, which is bounded by $\tilde{O}\left(m \beta^{-2} \epsilon^{-4}\right)$, up to poly-logarithmic factors. Thus, it follows that the operations ADDTERMINAL, INSERT and DeLETE can be implemented in $\tilde{O}(1)$ amortized update time.

\subsection{Dynamic All-Pairs Effective Resistance on Unweighted Graphs}

In this section we present one of the applications of our dynamic Schur complement data structure for unweighted graphs. Concretely, we design a dynamic algorithm that supports an intermixed sequence of edge insertions, deletion and pair-wise resistance queries, and returns a $(1 \pm \epsilon)$-approximation to all the resistance queries.

We start by reviewing two natural attempts for solving this problem.
- First, since spectral sparsifiers preserve effective resistances (Lemma 2.5), we could dynamically maintain a spectral sparsifier (Lemma 2.4), and then compute the $(s, t)$ effective resistance on this sparsifier. This leads to a data structure with poly $\left(\log n, \epsilon^{-1}\right)$ update time and $\tilde{O}\left(n \epsilon^{-2}\right)$ query time.

- Second, by the preservation of effective resistances under Schur complements (Fact 2.2), we could also utilize Schur complements to obtain a faster query time among a set of $\beta m$ terminals, $T$, for some reduction factor $\beta \in(0,1)$, at the expense of a slower update time. Specifically, after each edge update, we recompute an approximate Schur complement of the sparsifier onto $T$ in time $\tilde{O}\left(m \epsilon^{-2}\right)$ [16], after which each query takes $\tilde{O}\left(\beta m \epsilon^{-2}\right)$ time.

The first approach obtains sublinear update time, while the second approach gives sublinear query time. Our algorithm stems from combining these two methods, with the key additional observation being that adding more vertices to $T$ makes the Schur complement algorithm more local. Specifically, using Lemma 1.1 leads to a data-structure for dynamically maintaining all-pairs effective resistances.

Proof of Theorem 1.3. Let $\mathcal{D}(\tilde{H})$ denote the data structure that maintains a dynamic (sparse) Schur complement $\tilde{H}$ of $G$ (Lemma 1.1). Since $\mathcal{D}(\tilde{H})$ supports only up to $\beta m$ operations, we rebuild $\mathcal{D}(\tilde{H})$ on the current graph $G$ after such many operations. Note that the operations INSERT and Delete on $G$ are simply passed to $\mathcal{D}(\tilde{H})$. For processing the query operation EffectiveResistance $(s, t)$, we declare $s$ and $t$ terminals (using the operation AdDTERminal of $\mathcal{D}(\tilde{H})$ ), which ensures that they are both now contained in $\tilde{H}$. Finally, we compute the (approximate) effective resistance between $s$ and $t$ in $\tilde{H}$ using Lemma 2.1.

We now analyze the performance of our data-structure. Recall that the insertion or deletion of an edge in $G$ can be supported in $\tilde{O}(1)$ expected amortized time by $\mathcal{D}(\tilde{H})$. Since our data-structure is rebuilt every $\beta m$ operations, and rebuilding $\mathcal{D}(\tilde{H})$ can be implemented in $\tilde{O}\left(m \beta^{-2} \epsilon^{-4}\right)$, it follows that the amortized cost per edge insertion or deletion is

$$
\frac{\tilde{O}\left(m \beta^{-2} \epsilon^{-4}\right)}{\beta m}=\tilde{O}\left(\beta^{-3} \epsilon^{-4}\right) .
$$

The cost of any $(s, t)$ query is dominated by (1) the cost of declaring $s$ and $t$ terminals and (2) the cost of computing the $(s, t)$ effective resistance to $\epsilon$ accuracy on the graph $\tilde{H}$. Since (1) can be performed in $\tilde{O}(1)$ time, we only need to analyze (2). We do so by first giving a bound on the size of $T$. To this end, note that each of the $m$ edges in the current graph adds two vertices to $T$ with probability $\beta$ independently. By a Chernoff bound, the number of random augmentations added to $T$ is at most $2 \beta m$ with high probability. In addition, since $\mathcal{D}(\tilde{H})$ is rebuilt every $\beta m$ operations, the size of $T$ never exceeds $4 \beta m$ with high probability. The latter also bounds the size of $\tilde{H}$ by $\tilde{O}\left(\beta m \epsilon^{-2}\right)$ and gives that the query cost is $\tilde{O}\left(\beta m \epsilon^{-4}\right)$.

Combining the above bounds on the update and query time, we obtain the following trade-off

$$
\tilde{O}\left(\left(\beta m+\beta^{-3}\right) \epsilon^{-4}\right)
$$


which is minimized when $\beta=m^{-1 / 4}$, thus giving an expected amortized update and query time of

$$
\tilde{O}\left(m^{3 / 4} \epsilon^{-4}\right) \text {. }
$$

\subsection{Dynamic Schur Complement on Weighted Graphs}

In this section we briefly discuss an extension of Lemma 1.1 to weighted graphs at the cost of increasing the initialization time of the data-structure to $\tilde{O}\left(m \beta^{-4} \epsilon^{-4}\right)$ but keeping all the other guarantees the same. Similar to the previous subsection, we can use this result to maintain All-Pairs Effective Resistance in $\tilde{O}\left(m^{5 / 6} \epsilon^{-4}\right)$ update and query time. In fact, by running the data-structure on the output of a dynamic spectral sparsifier (Lemma 2.4), the running time per operation improves to $\tilde{O}\left(n^{5 / 6} \epsilon^{-6}\right)$.

While the extension of our dynamic Schur complement datastructure to weighted graphs builds upon the ideas we used in the unweighted case, there are a few obstacles that force us to introduce new components in our algorithm in order to make such an extension feasible. To illustrate, consider a path of constant length with edge weights alternating between 1 and $n^{10}$. Recall that the running time our data-structure depends on the speed at which random walks visit distinct edges in a graph. Due to the structure of the edge weights, a random walk in the path is expected to take $\Theta\left(n^{10}\right)$ steps before hitting a constant number of different edges. This shows that the naive generation of random walks in weighted graphs may be computationally prohibitive for our purposes.

To rectify the above issue, we make the important observation that it is not necessary to keep information for every single step of a random walk. Instead, it would suffice if we could perform the following step efficiently: (1) determine the step at which a random walk meets a new vertex (2) use this information to sample that vertex together with its corresponding edge and (3) estimate the weight of the random walk between any two given vertices. Bringing together these components allows us to generate random walks in weighted graphs much faster, and we next make this more precise.

Following the notation we used in the unweighted case, for an arbitrary vertex $v \in V$, a set of terminals $T \subseteq V$ and a parameter $\beta \in(0,1)$, a $\beta$-shorted walk with respect to $v$ and $T$ is a random walk that starts at $v$ and halts whenever $\Omega\left(\beta^{-1} \log n\right)$ distinct vertices have been hit, it reaches a vertex in $T$, or it has hit every edge in the connected component containing $v$. Our main contribution is summarized in the following lemma, whose proof is deferred to the full version of the paper.

LEMMA 4.8. Let $G=(V, E, w)$ be an undirected, weighted graph with polynomially bounded weights and $\epsilon>0$ an error parameter. Let $T \subseteq V$ be a set of terminals and $v \in V$ be an arbitrary vertex. Then there is an algorithm that generates a $\beta$-shorted random walk with respect to $v$ and $T$ and approximates its corresponding weight up to a $(1+\epsilon)$ multiplicative error in $\tilde{O}\left(\beta^{-4} \epsilon^{-2}\right)$ time.

\section{DYNAMIC LAPLACIAN SOLVER IN SUB-LINEAR TIME}

In this section we briefly describe the core idea behind the dynamic Laplacian solver that applies to unweighted, bounded degree graphs.
As described in Theorem 1.2, our goal is to design a data-structure that maintains a solution to the linear system $\mathbf{L x}=\mathbf{b}$ under updates to both the underlying graph and the demand vector vector $\mathbf{b}$ while being able to query a few entries of the solution vector.

Consider the dynamic Schur complement data-structure provided by Lemma 1.1. The prime difficulty of directly employing this data-structure to construct a dynamic solver is in handling the case where the demand vector $\mathbf{b}$ has a large support, i.e., a large number of non-zero entries. This is due to the fact that the standard reduction of appending all vertices corresponding to these non-zero entries to the Schur might lead to very inefficient running time guarantees when $\|\mathbf{b}\|_{0}=\Omega(n)$. We alleviate this by projection b onto the current set of terminals $T$ using the projection matrix $\mathbf{P}(T)$ (defined in Section 2) and showing that such an approximation to this projection can be maintained dynamically while introducing controllable error in the approximation guarantee of our solver. We summarize the guarantees of our dynamic projection routine in the following lemma and defer the implementation details and their analysis to the full version of this paper.

Lemma 5.1. Given an error parameter $\epsilon>0$, an unweighted unweighted bounded-degree $G=(V, E)$ with $n$ vertices, a vector $\mathbf{b} \in \mathbb{R}^{n}$ in the image of $\mathrm{L}$, a subset of terminal vertices $T^{\prime}$ and a parameter $\beta \in(0,1)$ such that $\left|T^{\prime}\right|=O(\beta m)$, there is a datastructure DYNAMICPRoJ $\left(G, T^{\prime}, \beta\right)$ for maintaining a vector $\tilde{\mathbf{b}}$ with $\|\tilde{\mathbf{b}}-\mathbf{P}(S) \mathbf{b}\|_{\mathbf{L}^{\dagger}} \leq \epsilon\|\mathbf{b}\|_{\mathbf{L}^{\dagger}}$ for some $T$ with $T^{\prime} \subseteq T,|T|=O(\beta m)$, while supporting at most $\beta^{3} m^{1 / 2} \epsilon(\text { poly } \log n)^{-1}$ operations in the following running times:

- INItialize $\left(G, T^{\prime}, \beta\right)$ : Initialize the data-struct. in Õ $(m)$ time.

- INSERT(u,v): Insert the edge $(u, v)$ to $G$ in $O(1)$ time while keeping $G$ bounded-degree.

- Delete $(u, v)$ : Delete the edge $(u, v)$ from $G$ in $O(1)$ time.

- CHANGE $\left(u, \mathbf{b}^{\prime}(u), v, \mathbf{b}^{\prime}(v)\right)$ : Change $\mathbf{b}(u)$ to $\mathbf{b}^{\prime}(u)$ and changes $\mathbf{b}(v)$ to $\mathbf{b}^{\prime}(v)$ while keeping $\mathbf{b}$ in the range of $\mathbf{L}$ in $O(1)$ time.

- AddTerminal $(u):$ Add u to T in O(1) time.

- QuerY(): Output the maintained $\tilde{\mathbf{b}}$ in $O(\beta m)$ time.

\section{ACKNOWLEDGMENTS}

We thank Daniel D. Sleator for helpful comments on an earlier version of this paper, and Xiaorui Sun for showing us a tight example on the maximum load of a vertex induced by random walks that hit distinct vertices.

The authors would also like to thank the anonymous referees for their valuable comments and helpful suggestions.

\section{REFERENCES}

[1] Ittai Abraham, Shiri Chechik, and Sebastian Krinninger. 2017. Fully dynamic all-pairs shortest paths with worst-case update-time revisited. In Symposium on Discrete Algorithms (SODA). 440-452.

[2] Ittai Abraham, David Durfee, Ioannis Koutis, Sebastian Krinninger, and Richard Peng. 2016. On Fully Dynamic Graph Sparsifiers. In Symposium on Foundations of Computer Science (FOCS). 335-344.

[3] Alexandr Andoni, Anupam Gupta, and Robert Krauthgamer. 2014. Towards $(1+\epsilon)$-approximate flow sparsifiers. In Symposium on Discrete algorithms (SODA). 279-293.

[4] Alexandr Andoni, Robert Krauthgamer, and Yosef Pogrow. 2019. On Solving Linear Systems in Sublinear Time. In Innovations in Theoretical Computer Science Conference (ITCS). 3:1-3:19.

[5] Greg Barnes and Uriel Feige. 1996. Short Random Walks on Graphs. SIAM fournal on Discrete Mathemathics 9, 1 (1996), 19-28. 
[6] Surender Baswana, Manoj Gupta, and Sandeep Sen. 2015. Fully Dynamic Maximal Matching in $O(\log n)$ Update Time. SIAM f. Comput. 44, 1 (2015), 88-113. Announced at FOCS'11.

[7] Surender Baswana, Sumeet Khurana, and Soumojit Sarkar. 2012. Fully dynamic randomized algorithms for graph spanners. ACM Transactions on Algorithms (TALG) 8, 4 (2012), 35.

[8] Joshua Batson, Daniel A. Spielman, Nikhil Srivastava, and Shang-Hua Teng. 2013 Spectral sparsification of graphs: theory and algorithms. Commun. ACM 56, 8 (Aug. 2013), 87-94. https://doi.org/10.1145/2492007.2492029

[9] Andras A. Benczur and David R. Karger. 1996. Approximating s-t minimum cuts in $\tilde{O}\left(n^{2}\right)$ time. In Symposium on Theory of computing (STOC). 47-55.

[10] Aaron Bernstein and Shiri Chechik. 2016. Deterministic decremental single source shortest paths: beyond the $\mathrm{O}(\mathrm{mn})$ bound. In Symposium on Theory of Computing (STOC). 389-397.

[11] Sayan Bhattacharya, Monika Henzinger, and Danupon Nanongkai. 2016. New deterministic approximation algorithms for fully dynamic matching. In Symposium on Theory of Computing (STOC). 398-411.

[12] Moses Charikar, Tom Leighton, Shi Li, and Ankur Moitra. 2010. Vertex sparsifier and abstract rounding algorithms. In Symposium on Foundations of Computer Science (FOCS). 265-274.

[13] Dehua Cheng, Yu Cheng, Yan Liu, Richard Peng, and Shang-Hua Teng. 2015. Efficient Sampling for Gaussian Graphical Models via Spectral Sparsification. Conference on Learning Theory (COLT) (2015), 364-390.

[14] Camil Demetrescu and Giuseppe F. Italiano. 2004. A new approach to dynamic all pairs shortest paths. F. ACM 51, 6 (2004), 968-992.

[15] Florian Dörfler and Francesco Bullo. 2013. Kron Reduction of Graphs With Applications to Electrical Networks. IEEE Trans. on Circuits and Systems 60-I, 1 (2013), 150-163.

[16] David Durfee, Rasmus Kyng, John Peebles, Anup B Rao, and Sushant Sachdeva 2017. Sampling random spanning trees faster than matrix multiplication. In Symposium on Theory of Computing (STOC). 730-742.

[17] David Durfee, John Peebles, Richard Peng, and Anup B. Rao. 2017. DeterminantPreserving Sparsification of SDDM Matrices with Applications to Counting and Sampling Spanning Trees. In Symposium on Foundations of Computer Science (FOCS). 926-937.

[18] David Eppstein. 1991. Offline algorithms for dynamic minimum spanning tree problems. In Workshop on Algorithms and Data Structures (WADS). 392-399.

[19] David Eppstein, Zvi Galil, Giuseppe F Italiano, and Amnon Nissenzweig. 1997. Sparsification: a technique for speeding up dynamic graph algorithms. $\mathcal{F}$. ACM 44, 5 (1997), 669-696. Announced at FOCS'92.

[20] Jittat Fakcharoenphol, Satish Rao, and Kunal Talwar. 2004. A tight bound on approximating arbitrary metrics by tree metrics. f. Comput. Syst. Sci. 69, 3 (2004), 485-497.

[21] Greg N Frederickson. 1985. Data structures for on-line updating of minimum spanning trees, with applications. SIAM f. Comput. 14, 4 (1985), 781-798. Announced at STOC'84.

[22] Gramoz Goranci, Monika Henzinger, and Pan Peng. 2017. The Power of Vertex Sparsifiers in Dynamic Graph Algorithms. In European Symposium on Algorithms (ESA). 45:1-45:14

[23] Gramoz Goranci, Monika Henzinger, and Pan Peng. 2018. Dynamic Effective Resistances and Approximate Schur Complement on Separable Graphs. In European Symposium on Algorithms (ESA). 40:1-40:15.

[24] Gramoz Goranci, Monika Henzinger, and Mikkel Thorup. 2016. Incremental Exact Min-Cut in Poly-logarithmic Amortized Update Time. In European Symposium on Algorithms (ESA). 46:1-46:17.

[25] Gramoz Goranci and Sebastian Krinninger. 2018. Dynamic Low-Stretch Trees via Dynamic Low-Diameter Decompositions. CoRR abs/1804.04928 (2018). Available at: http://arxiv.org/abs/1804.04928.

[26] Manoj Gupta and Richard Peng. 2013. Fully dynamic $(1+\epsilon)$-approximate match ings. In Symposium on Foundations of Computer Science (FOCS). 548-557.

[27] Monika Henzinger, Sebastian Krinninger, and Danupon Nanongkai. 2014. Decremental single-source shortest paths on undirected graphs in near-linear total update time. In Symposium on Foundations of Computer Science (FOCS). 146-155.

[28] Monika Henzinger, Sebastian Krinninger, and Danupon Nanongkai. 2016. Dynamic Approximate All-Pairs Shortest Paths: Breaking the $\mathrm{O}(\mathrm{mn})$ Barrier and Derandomization. SIAM f. Comput. 45, 3 (2016), 947-1006. Announced at FOCS'13.

[29] Monika Rauch Henzinger. 1997. A static 2-approximation algorithm for vertex connectivity and incremental approximation algorithms for edge and vertex connectivity. Journal of Algorithms 24, 1 (1997), 194-220.

[30] Jacob Holm, Kristian De Lichtenberg, and Mikkel Thorup. 2001. Poly-logarithmic deterministic fully-dynamic algorithms for connectivity, minimum spanning tree, 2-edge, and biconnectivity. F. ACM 48, 4 (2001), 723-760. Announced at STOC'98.

[31] Jacob Holm, Eva Rotenberg, and Christian Wulff-Nilsen. 2015. Faster Fully Dynamic Minimum Spanning Forest. In European Symposium on Algorithms (ESA). 742-753.
[32] Bruce M Kapron, Valerie King, and Ben Mountjoy. 2013. Dynamic graph connectivity in polylogarithmic worst case time. In Symposium on Discrete Algorithms (SODA). 1131-1142.

[33] Jonathan A. Kelner, Yin Tat Lee, Lorenzo Orecchia, and Aaron Sidford. 2014. An Almost-Linear-Time Algorithm for Approximate Max Flow in Undirected Graphs, and its Multicommodity Generalizations. In Symposium on Discrete Algorithms (SODA). 217-226.

[34] Ioannis Koutis, Gary L. Miller, and Richard Peng. 2011. A Nearly-m log n Time Solver for SDD Linear Systems. In Symposium on Foundations of Computer Science (FOCS). 590-598.

[35] Robert Krauthgamer and Inbal Rika. 2013. Mimicking networks and succinct representations of terminal cuts. In Symposium on Discrete algorithms (SODA). 1789-1799.

[36] Rasmus Kyng, Yin Tat Lee, Richard Peng, Sushant Sachdeva, and Daniel A Spielman. 2016. Sparsified Cholesky and multigrid solvers for connection laplacians. In Symposium on Theory of Computing (STOC). 842-850.

[37] Rasmus Kyng and Sushant Sachdeva. 2016. Approximate gaussian elimination for laplacians-fast, sparse, and simple. In Symposium on Foundations of Computer Science (FOCS). 573-582.

[38] Jakub Lacki and Piotr Sankowski. 2011. Min-cuts and shortest cycles in planar graphs in O (n loglogn) time. In European Symposium on Algorithms (ESA). 155166.

[39] Huan Li and Zhongzhi Zhang. 2018. Kirchhoff Index As a Measure of Edge Centrality in Weighted Networks: Nearly Linear Time Algorithms. In Symposium on Discrete Algorithms (SODA). 2377-2396.

[40] David Liben-Nowell and Jon M. Kleinberg. 2003. The link prediction problem for social networks. In International Conference on Information and Knowledge Management (CIKM). 556-559.

[41] Andreas Loukas. 2018. Graph reduction by local variation. CoRR abs/1808.10650 (2018).

[42] Andreas Loukas and Pierre Vandergheynst. 2018. Spectrally Approximating Large Graphs with Smaller Graphs. In ICML ( $7 M L R$ Workshop and Conference Proceedings), Vol. 80. JMLR.org, 3243-3252.

[43] Aleksander Madry. 2010. Fast approximation algorithms for cut-based problems in undirected graphs. In Symposium on Foundations of Computer Science (FOCS). 245-254.

[44] Aleksander Madry, Damian Straszak, and Jakub Tarnawski. 2015. Fast Generation of Random Spanning Trees and the Effective Resistance Metric. In Symposium on Discrete Algorithms (SODA). 2019-2036.

[45] Konstantin Makarychev and Yury Makarychev. 2010. Metric Extension Operators, Vertex Sparsifiers and Lipschitz Extendability. In Symposium on Foundations of Computer Science (FOCS). 255-264.

[46] Ankur Moitra. 2009. Approximation Algorithms for Multicommodity-Type Problems with Guarantees Independent of the Graph Size. In Symposium on Foundations of Computer Science (FOCS). 3-12.

[47] Danupon Nanongkai and Thatchaphol Saranurak. 2017. Dynamic spanning forest with worst-case update time: adaptive, Las Vegas, and $O\left(n^{1 / 2-\epsilon}\right)$-time. In Symposium on Theory of Computing (STOC). 1122-1129.

[48] Danupon Nanongkai, Thatchaphol Saranurak, and Christian Wulff-Nilsen. 2017. Dynamic Minimum Spanning Forest with Subpolynomial Worst-Case Update Time. In Symposium on Foundations of Computer Science (FOCS). 950-961.

[49] Ofer Neiman and Shay Solomon. 2016. Simple Deterministic Algorithms for Fully Dynamic Maximal Matching. ACM Trans. Algorithms 12, 1 (2016), 7:1-7:15. Announced at STOC'13.

[50] Krzysztof Onak and Ronitt Rubinfeld. 2010. Maintaining a large matching and a small vertex cover. In Symposium on Theory of computing (STOC). 457-464.

[51] David Peleg and Alejandro A Schäffer. 1989. Graph spanners. Journal of graph theory 13, 1 (1989), 99-116.

[52] Richard Peng, Bryce Sandlund, and Daniel Dominic Sleator. 2017. Offline Dynamic Higher Connectivity. CoRR abs/1708.03812 (2017). Available at: http://arxiv.org/abs/1708.03812.

[53] Richard Peng and Daniel A. Spielman. 2014. An efficient parallel solver for SDD linear systems. In Symposium on Theory of Computing (STOC). 333-342.

[54] Harald Räcke. 2008. Optimal hierarchical decompositions for congestion minimization in networks. In Symposium on Theory of computing (STOC). 255-264.

[55] Piotr Sankowski. 2004. Dynamic Transitive Closure via Dynamic Matrix Inverse (Extended Abstract). In Symposium on Foundations of Computer Science (FOCS). 509-517.

[56] Aaron Schild. 2018. An almost-linear time algorithm for uniform random spanning tree generation. In Symposium on Theory of Computing (STOC). 214-227.

[57] Aaron Schild, Satish Rao, and Nikhil Srivastava. 2018. Localization of Electrical Flows. In Symposium on Discrete Algorithms (SODA). 1577-1584.

[58] Jonah Sherman. 2013. Nearly Maximum Flows in Nearly Linear Time. In Symposium on Foundations of Computer Science (FOCS). 263-269.

[59] Shay Solomon. 2016. Fully Dynamic Maximal Matching in Constant Update Time. In Foundations of Computer Science (FOCS). 325-334. 
[60] Daniel Spielman and Nikhil Srivastava. 2011. Graph Sparsification by Effective Resistances. SIAM F. Comput. 40, 6 (2011), 1913-1926.

[61] D. Spielman and S. Teng. 2011. Spectral Sparsification of Graphs. SIAM 7. Comput. 40, 4 (2011), 981-1025.

[62] D. Spielman and S. Teng. 2014. Nearly Linear Time Algorithms for Preconditioning and Solving Symmetric, Diagonally Dominant Linear Systems. SIAM 7 Matrix Anal. Appl. 35, 3 (2014), 835-885.

[63] Daniel A. Spielman. 2010. Algorithms, Graph Theory, and Linear Equations in Laplacian Matrices. In Proceedings of the International Congress of Mathematicians.
[64] Shang-Hua Teng. 2010. The Laplacian Paradigm: Emerging Algorithms for Massive Graphs. In Theory and Applications of Models of Computation. 2-14.

[65] Mikkel Thorup. 2007. Fully-dynamic min-cut. Combinatorica 27, 1 (2007), 91-127. Announced at STOC'01.

[66] Tal Wagner, Sudipto Guha, Shiva Prasad Kasiviswanathan, and Nina Mishra. 2018. Semi-Supervised Learning on Data Streams via Temporal Label Propagation. In International Conference on Machine Learning (ICML). 5082-5091.

[67] Christian Wulff-Nilsen. 2017. Fully-dynamic minimum spanning forest with improved worst-case update time. In Symposium on Theory of Computing (STOC). $1130-1143$ 